\title{
Achados de neuroimagem no transtorno obsessivo-compulsivo
}

\author{
Acioly Luiz T de Lacerdaa, Paulo Dalgalarrondo e Edwaldo Eduardo Camargo ${ }^{b}$

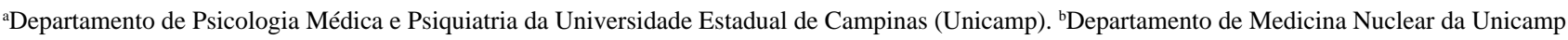

\section{Introdução}

O transtorno obsessivo-compulsivo (TOC) acomete cerca de $2 \%$ da população geral e é caracterizado pela presença de pensamentos e/ou comportamentos repetitivos e intrusivos, que causam desconforto importante.

Com descrições datando da Idade Média, o TOC teve a sua etiologia atribuída aos mais variados fatores, desde "possessão demoníaca" até conflitos emocionais da infância (Freud, no início do século XX), prejuízo na circulação cerebral (Ball, no final do século XIX) e danos neurais subjacentes (Esquirol, na primeira metade do século XIX).

Os fenômenos obsessivos, no entanto, podem ocorrer em pessoas normais, que facilmente se desvencilham destes, o que não acontece com o indivíduo com TOC. O caráter repetitivo e rígido dos sintomas obsessivo-compulsivos há muito chamou a atenção dos estudiosos, sugerindo um controle cognitivo inadequado, assim como relações entre tal sintomatologia e outros comportamentos estereotipados que se desenvolvem em doenças neurológicas.

O TOC vem sendo reconhecido de modo crescente como uma condição neuropsiquiátrica, pois existem fortes evidências apontando para uma disfunção cerebral subjacente. ${ }^{1}$ Tal perspectiva é apoiada pelos seguintes achados:

- a ocorrência de sintomas obsessivo-compulsivos em diversas doenças neurológicas, incluindo a síndrome de Gilles de la Tourette, Parkinson pós-encefalítico, coréia de Sydenham e epilepsia do lobo temporal;

- a resposta a terapias biológicas, como o uso de inibidores potentes da recaptura da serotonina, e psicocirurgia, em alguns casos refratários;

- o relato da presença de sinais neurológicos leves (presentes em até $90 \%$ dos pacientes) e de alterações de potenciais evocados corticais nesses pacientes;

- estudos neuropsicológicos, apontando para um distúrbio das funções executivas (pré-frontais), das funções não-verbais e das funções visuoespaciais, todas envolvendo o circuito fronto-estriato-tálamo-cortical, quando em comparação com controles saudáveis;

- estudos epidemiológicos, mostrando uma prevalência aumentada de TOC em parentes de primeiro grau de indivíduos acometidos, quando comparados à prevalência da população geral, indicando um importante componente genético para o transtorno.

$\mathrm{O}$ estudos de neuroimagem estrutural e funcional no TOC vieram reforçar tais evidências, inclusive possibilitando a teorização acerca da fisiopatologia desse transtorno, a partir das conclusões dos seus mais variados desenhos de estudo, os quais são temas do presente artigo.

\section{Neuroimagem estrutural}

Estes estudos - embora com uma menor probabilidade de apresentarem achados mais consistentes, já que o próprio avanço da psicofarmacologia tem sugerido que os transtornos psiquiátricos decorrem muito provavelmente de alterações funcionais, em contraposição a alterações anatômicas - podem esclarecer se essas disfunções apresentam uma alteração anatômica subjacente ou se são primárias.

\section{Estudos com imagem por ressonância magnética (IRM)}

Em função da tomografia computadorizada (TC) ter sido utilizada em poucos estudos, com resultados contraditórios e, principalmente, por ser mais limitada do que a IRM, esses estudos não foram revisados no presente artigo. Assim como os estudos com TC, os com IRM também são contraditórios e de conclusões pouco robustas.

Comparada à TC, a IRM apresenta melhor resolução espacial, distinção entre substâncias branca e cinzenta e visualização de estruturas neuroanatômicas em vários planos, de modo a possibilitar reconstruções tridimensionais de estruturas anatômicas e o cálculo de seus volumes.

Jenike et al relataram uma redução da substância branca e aumento do volume cortical no TOC. ${ }^{2}$ Rosenberg et al encontraram um alargamento do terceiro ventrículo em crianças com TOC (achados difusos). ${ }^{3}$

Outros estudos descrevem alterações localizadas, como aumento ${ }^{4}$ ou redução ${ }^{5}$ do volume dos núcleos caudados; redução do volume do corpo estriatal; ${ }^{3}$ redução do volume das amígdalas cerebrais e das regiões órbito-frontais; ${ }^{6}$ aumento dos volumes talâmicos em crianças sem história de tratamento prévio, seguindo-se de uma redução após tratamento com paroxetina. ${ }^{7}$

Aylward et al, ${ }^{8}$ Kellner et al ${ }^{9} \mathrm{e}$ Bartha et $\mathrm{al}^{10}$ não encontraram diferenças estruturais em relação aos controles saudáveis, quando mensurados os núcleos caudados.

A Tabela 1 sumariza os resultados destes estudos. Os estudos de neuroimagem estrutural não puderam, até o momento, identificar padrões homogêneos de alterações, localizadas ou difusas, nem alterações específicas em relação a outros transtornos psiquiátricos ou neuropsiquiátricos. ${ }^{1}$ 


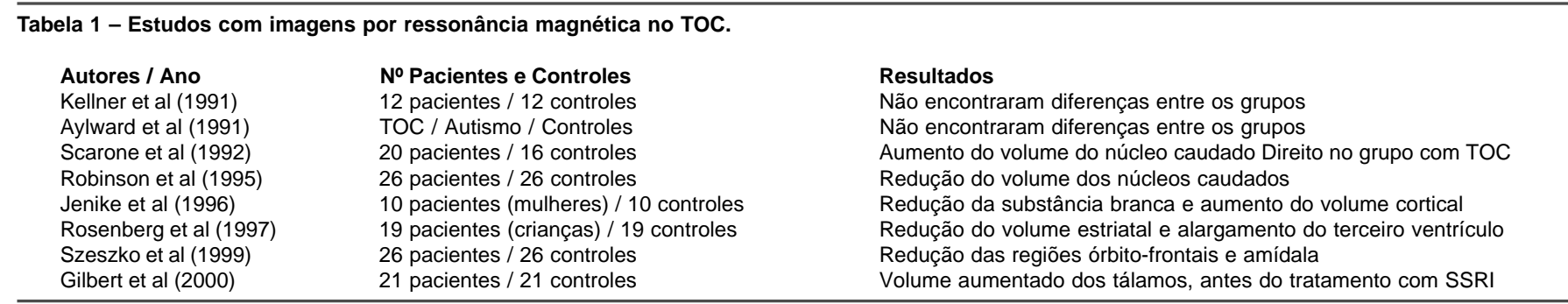

\section{Neuroimagem funcional}

Para os que se interessam pela fisiopatologia dos transtornos psiquiátricos, os últimos anos têm testemunhado uma mudança marcante de paradigma. Da confiança depositada nos modelos animais de psicopatologia, com todas as suas negligências, para a neuroendocrinologia, com as suas armadilhas, e para os estudos postmortem com suas dificuldades inerentes, o campo tem evoluído para o uso de técnicas multidisciplinares, dentre as quais a neuroimagem funcional destacase como uma das mais promissoras, embora pouco conhecida. ${ }^{11}$

Estão resumidos, a seguir, os achados nos pacientes com TOC quando do uso das técnicas chamadas "the big four" da neuroimagem funcional, ou seja, espectroscopia por ressonância magnética, imagem por ressonância magnética funcional (IMRf), PET (positron emission tomography - tomografia por emissão de pósitrons) e SPECT (single photon emission computed tomography - tomografia computadorizada por emissão de fótons únicos. A grande maioria dos estudos utilizaram as duas últimas técnicas.

\section{Espectroscopia por ressonância magnética}

A espectroscopia é um meio não-invasivo e seguro (isento de radioatividade), que possibilita o estudo do metabolismo e da neuroquímica cerebral, abrindo, portanto, o interminável veio científico da neuroquímica cerebral nos transtornos mentais.

Ebert et al, estudando 12 pacientes com TOC e seis controles, encontraram níveis significativamente reduzidos de Nacetil-aspartato (NAA) no estriado direito e cíngulo anterior; o último achado correlacionou-se negativamente com a gravidade do transtorno. ${ }^{12}$ O NAA é um possível marcador neuronal que se encontra reduzido em doenças que envolvem perda neuronal, lesão ou redução tecidual.

Ohara et al, avaliando os picos de NAA, creatina ( $\mathrm{Cr}$ ) e colina (Cho), não encontraram diferença significativa quando estudado o núcleo lenticular, concluindo que há uma viabilidade neuronal normal nessa estrutura. ${ }^{13}$

Rosenberg et al, avaliando 11 crianças sem tratamento prévio, encontraram concentrações glutamatérgicas significativamente aumentadas nos núcleos caudados, quando comparadas a controles saudáveis. ${ }^{14}$ Registraram, também, uma redução significativa das concentrações deste neurotransmissor nos núcleos caudados após o tratamento com paroxetina, o que correlacionou-se com a redução dos sintomas.

\section{Imagem por ressonância magnética funcional}

A ressonância magnética funcional constitui uma técnica sensível às mudanças locais da concentração de deoxi- hemoglobina, de modo que, quando há o aumento do fluxo sangüíneo cerebral em determinada região com maior atividade, a oferta de oxigênio supera o consumo com uma aparente redução da deoxi-hemoglobina.

Breiter et al observaram, quando da provocação sintomática, uma ativação para $70 \%$ ou mais dos pacientes nas seguintes regiões: órbito-frontal medial, frontal lateral, temporal anterior, anterior do cíngulo, córtex da ínsula, núcleo caudado, núcleo lenticulado e amígdala cerebral. ${ }^{15}$ Os controles não exibiram ativação significativa em nenhuma das regiões de interesse. Os autores concluíram que os resultados são compatíveis com os encontrados com outras técnicas de neuroimagem funcional e ressaltam ativações mais proeminentes nos sistemas límbico e paralímbico no estudo.

Levine et al, comparando subgrupos de pacientes esquizofrênicos com intensa sintomatologia obsessivo-compulsiva com os que não apresentavam esse quadro, observaram que um subgrupo dos primeiros apresentava uma correlação negativa entre a intensidade da sintomatologia e a ativação do córtex pré-frontal esquerdo, sugerindo a participação dessa região em modelo fisiopatológico do TOC. ${ }^{16}$

Pujol et al compararam um grupo de pacientes com controles saudáveis durante a execução de uma tarefa de geração de palavras. ${ }^{17}$ Observaram que os pacientes apresentaram uma ativação no córtex frontal significativamente maior durante a tarefa e uma supressão prejudicada desta ativação durante o período de repouso. Ambos os achados correlacionaram-se significativamente com a intensidade dos sintomas.

\section{Positron emission tomography (PET)}

Quanto ao desenho, podemos dividir os diversos estudos que utilizaram PET na avaliação de pacientes com TOC em grandes grupos (Tabela 2).

\section{Estudos em repouso}

Foram sete os estudos publicados, comparando pacientes com TOC a controles normais, em repouso. Em seis desses estudos, demonstrou-se um aumento do metabolismo de glicose no córtex órbito-frontal, ao passo que apenas um estudo apresentou um achado oposto na mesma região. ${ }^{18}$

Anormalidades funcionais nos núcleos caudados mostraramse menos consistentes. Estudos com PET apontaram aumento, ${ }^{19}$ redução ${ }^{18}$ ou ausência de alteração no consumo de glicose nesta região. ${ }^{20}$

Dois estudos relatam um aumento no metabolismo no giro do cíngulo, ao passo que, em outros cinco estudos, não foram observadas alterações do metabolismo nesta região, quando 


\begin{tabular}{|c|c|c|}
\hline Autor / Ano & Número de pacientes e Controles & Resultados \\
\hline Baxter et al (1987) & 14 pacientes / 14 controles & Elevado metabolismo no giro orbital esquerdo \\
\hline Martinot et al (1990) & 16 pacientes / 8 controles & $\begin{array}{l}\text { Taxa de utilização de glicose menor no córtex cerebral como um todo e no } \\
\text { córtex pré-frontal dos pacientes }\end{array}$ \\
\hline Swedo et al (1992) & 13 pacientes / Nenhum controle & $\begin{array}{l}\text { Significativa redução do metabolismo de glicose em ambas as regiões órbito- } \\
\text { frontais, } 1 \text { ano após tratamento com ISRS }\end{array}$ \\
\hline Baxter et al (1992) & $\begin{array}{l}.10 \text { pacientes submetidos a tratamento com fluoxetina/ } \\
.10 \text { pacientes submetidos a terapia comportamental }\end{array}$ & $\begin{array}{l}\text { Redução do metabolismo da cabeça do núcleo caudado direito em pacientes } \\
\text { respondedores de ambos os grupos; Metabolismo órbito-frontal } D \\
\text { correlacionado positivamente com } n \text {. caudado e tálamo homolaterais em } \\
\text { respondedores. }\end{array}$ \\
\hline Rauch (1994) & 8 pacientes & $\begin{array}{l}\text { Aumento do metabolismo de glicose durante provocação de sintomas nas } \\
\text { seguintes regiões: } n \text {. Caudado } D \text {, córtex do cíngulo } E \text {, córtex órbito-frontal } \\
\text { bilateral. }\end{array}$ \\
\hline Schwartz et al (1996) & 9 pacientes & $\begin{array}{l}\text { Os pacientes respondedores à Terapia Comportamental tiveram uma } \\
\text { significativa redução no metabolismo de glicose de ambos os } \mathrm{n} \text {. Caudados }\end{array}$ \\
\hline
\end{tabular}

comparados a controles saudáveis. ${ }^{21}$

Dos sete estudos, apenas um demonstrou um aumento significativo do metabolismo nos tálamos, não havendo diferença significativa nos outros. ${ }^{22}$

\section{Estudos pré e pós-tratamento}

Foram realizados sete estudos comparando o metabolismo cerebral de pacientes com TOC, antes e após tratamento com inibidores potentes de recaptura da serotonina, terapia cognitivo-comportamental ou capsulotomia. Todos esses estudos encontraram redução significativa do metabolismo cerebral em uma ou mais áreas que compõem o circuito pré-frontalestriado-tálamo-cortical. Em seis deles, observou-se uma redução nas regiões órbito-frontais; cinco demonstraram redução do metabolismo nos núcleos caudados; dois estudos, nos tálamos; e apenas um estudo demonstrou redução do metabolismo no giro do cíngulo, após o tratamento. ${ }^{1}$

\section{Estudos com provocação sintomática}

Apenas dois estudos foram realizados com este desenho. Em ambos, observou-se um aumento do metabolismo nas regiões órbito-frontais, estriado, giro do cíngulo e tálamos. Um dos estudos, além dessas regiões, relatou aumento do metabolismo no hipocampo e no globo pálido, quando houve a provocação sintomática. $^{23}$

\section{Single photon emission computed tomography (SPECT)}

\section{Estudos em repouso}

Sete foram os estudos com SPECT em pacientes com TOC, comparados a controles saudáveis em repouso (Tabela 3). ${ }^{1,24-29}$ Em três deles houve um aumento do fluxo sangüíneo cerebral regional (FSCr) nas regiões frontais. Três estudos mostraram uma redução do FSCr nos núcleos da base.

Dois estudo publicados pelos autores do presente estudo relatam alterações do FSCr nas regiões frontais direitas (superior, inferior e dorsolateral), seja de redução ${ }^{29}$ ou de aumento. ${ }^{1}$ Relatam também redução do FSCr no córtex anterior dorsal es- querdo do cíngulo no primeiro estudo e aumento do FSCr nos tálamos, no segundo estudo, com tendência a normalização após tratamento com clomipramina (Figuras 1, 2 e 3).

\section{Estudos com “desafios farmacológicos”}

Nos dois estudos de "desafio farmacológico", observou-se um aumento do FSCr no córtex cerebral globalmente (uso de $\mathrm{m}$-CPP), assim como uma correlação negativa entre o $\mathrm{FSCr}$ nas regiões frontais e a exacerbação sintomática com o uso do sumatriptano (dose única de $100 \mathrm{mg}, 90$ minutos antes da injeção do 99mTc HMPAO). ${ }^{30}$

\section{Estudos pré e pós tratamento}

Em dois estudos realizados antes e após o tratamento com inibidor potente de recaptura da serotonina, houve uma significativa redução na razão do $\mathrm{FSCr}$ entre as regiões frontais mediais/córtex e no córtex, após o tratamento. ${ }^{25,31}$

\section{Conclusão}

Os achados de neuroimagem mais consistentes no TOC referem-se, em ordem decrescente de importância, a alterações nas regiões órbito-frontais, núcleos da base, giro do cíngulo e tálamos. Portanto, apontam quase invariavelmente para alterações nas regiões que compõem o circuito pré-frontal-estriadotálamo-cortical.

O fato deste circuito ter papel fundamental no processamento cortical de informações, no início de respostas comportamentais ou cognitivas, aliado às evidências clínicas oriundas tanto de doenças neurológicas (por exemplo, perseveração) como do próprio TOC, faz pensar que as técnicas de neuroimagem propiciarão um salto qualitativo no entendimento fisiopatológico deste transtorno.

As conseqüências desse avanço serão marcantes, tanto no que se refere a um refinamento na classificação, quanto de categoria (baseada simplesmente na expressão sintomatológica); pode evoluir para uma classificação fisiopatológica quanto ao tratamento deste transtorno, que ainda desafia as neurociências, no final do século XX. 


\section{Referências}

1. Lacerda ALT. Transtorno obsessivo compulsivo: um estudo psicopatológico, neuropsicológico e de fluxo sangüíneo cerebral regional (HMPAO-Tc99m) [tese]. Campinas: Universidade Estadual de Campinas; 2000.

2. Jenike MA, Breiter HC, Baer L, Kennedy DN, Savage CR, Olivares MJ, et al. Cerebral structural abnormalities in obsessive-compulsive disorder: a quantitative morphometric magnetic resonance imaging study. Arch Gen Psychiatry 1996;53:625-32.

3. Rosenberg DR, Keshavan MS, O'Hearn KM, Dick EL, Bagwell WW, Seymour AB, et al. Frontostriatal measurement in treatmentnaive children with obsessive-compulsive disorder. Arch Gen Psychiatry 1997;54:824-30.

4. Scarone S, Colombo C, Livian S, Abbruzzese M, Ronchi P, Locatelli $\mathrm{M}$, et al. Increased right caudate nucleus size in obsessivecompulsive disorder: detection with magnetic resonance imaging. Psychiatry Res Neuroimaging 1992;45:115-21.

5. Robinson D, Wu H, Munne RA, Ashtari M, Alvir JM, Lerner G, et al. Reduced caudate nucleus volume in obsessive-compulsive disorder. Arch Gen Psychiatry 1995;52:393-8.

6. Szeszko PR, Robinson D, Alvir JM, Bilder RM, Lencz T, Ashtari $\mathrm{M}$, et al. Orbital frontal and amygdala volume reductions in obsessive-compulsive disorder. Arch Gen Psychiatry. 1999;56:9139.

7. Gilbert AR, Moore GJ, Keshavan MS, Paulson LA, Narula V, Mac Master FP, et al. Decrease in thalamic volumes of pediatric patients with obsessive-compulsive disorder who are taking paroxetine. Arch Gen Psychiatry. 2000;57:449-56.

8. Aylward EH, Schwartz J, Machlin S, Pearlson G. Bicaudate ratio as a measure of caudate volume on MR images. AJNR Am J Neuroradiol 1991;12:1217-22.

9. Kellner CH, Jolley RR, Holgate RC, Austin L, Lydiard RB, Laraia $\mathrm{M}$, et al. Brain MRI in obsessive-compulsive disorder. Psychiatry Res 1991;36:45-9.

10. Bartha R, Stein MB, Williamson PC, Drost DJ, Neufeld RW, Carr $\mathrm{TJ}$, et al. A short echo $1 \mathrm{H}$ spectroscopy and volumetric MRI study of the corpus striatum in patients with obsessive-compulsive disorder and comparison subjects. Am J Psychiatry 1998; 155:1584-91.

11. Nemeroff CB, Kilts CD, Berns GS. Functional Brain Imaging: twenty-first century phrenology or psyuchobiological advance for the millenium? [editorial]. Am J Psychiatry 1999;156:671-3.

12. Ebert D, Speck O, Konig A, Berger M, Hennig J, Hohagen F. $1 \mathrm{H}-$ magnetic resonance spectroscopy in obsessive-compulsive disorder: evidence for neuronal loss in the cingulate gyrus and the right striatum. Psychiatry Res 1997;74:173-6.

13. Ohara K, Isoda H, Suzuki Y, Takehara Y, Ochiai M, Takeda H, et al. Proton magnetic resonance spectroscopy of lenticular nuclei in obsessive-compulsive disorder. Psychiatry Res 1999;92:83-91.

14. Rosenberg DR, MacMaster FP, Keshavan MS, Fitzgerald KD, Stewart CM, Moore GJ. Decrease in caudate glutamatergic concentrations in pediatric obsessive-compulsive disorder patients taking paroxetine. J Am Acad Child Adolesc Psychiatry 2000;39:1096-103.

15. Breiter HC, Rauch SL, Kwong KK, Baker JR, Weisskoff RM, Kennedy DN, et al. Functioning magnetic resonance imaging of symptom provocation in obsessive-compulsive disorder. Arch Gen Psychiatry 1996;49:545-606.

16. Levine JB, Gruber SA, Baird AA, Yurgelun-Todd D. Obsessivecompulsive disorder among schizophrenic patients: an exploratory study using functional magnetic resonance imaging data. Compr Psychiatry 1998;39(5):308-11.
17. Pujol J, Torres L, Deus J, Cardoner N, Pifarre J, Capdevila A, et al. Functional magnetic resonance imaging study of frontal lobe activation during word generation in obsessive-compulsive disorder. Biol Psychiatry 1999;45(7):891-7.

18. Martinot JL, Allilaire JF, Mazoyer BM, Hantouche E, Huret JD, LegautDemare F, et al. Obsessive-compulsive disorder: a clinical, neuropsychological and positron emission tomography study. Acta Psychiatr Scand 1990;82:233-42.

19. Baxter LR, Phelps ME, Mazziotta JC, Guze BH, Schwartz JM, Selin CE. Local cerebral glucose metabolic rates in obsessive-compulsive disorder. Arch Gen Psychiatry 1987;44:211-8.

20. Swedo SE, Pietrini P, Leonard HL, Schapiro MB, Rettew DC, Goldberger EL, et al. Cerebral glucose metabolism in childhood-onset obsessivecompulsive disorder: revisualization during pharmacotherapy. Arch Gen Psychiatry 1992;49:690-4.

21. Baxter LR, Schwartz JM, Bergmann KS, Szuba MP, Guze BH, Mazziotta JC, et al. Caudate glucose metabolic rate changes with both drug and behaviour therapy for obsessive-compulsive disorder. Arch Gen Psychiatry 1992;49:690-4.

22. Perani D, Colombo C, Bressi S, Bonfanti A, Grassi F, Scarone S, et al. [18F]FDG PET study in obsessive-compulsive disorder: a clinical/ metabolic correlation study after treatment. Br J Psychiatry 1995;166:244-50.

23. Rauch SL, Jenike MA, Alpert NM, Baer L, Breiter HC, Savage CR, et al. Regional cerebral blood flow measured during symptom provocation in obsessive-compulsive disorder: a PET study of implicit sequence learning. Arch Gen Psychiatry 1994;51:62-70.

24. Machlin SR, Harris GJ, Pearlson GD, Hoehn-Saric R, Jeffery P, Camargo EE. Elevated medial-frontal cerebral blood flow in obsessive-compulsive patients: a SPECT study. Am J Psychiatry 1991;148:1240-2.

25. Rubin RT, Villanueva-Meyer J, Ananth J, Trajmar PG, Mena I. Regional xenon 133 cerebral blood flow and cerebral technetium 99m HMPAO uptake in unmedicated patients with obsessive-compulsive disorder and matched normal control subjects. Determination by high-resolution single-proton emission cumputed tomography. Arch Gen Psychiatry 1992;49:695-702.

26. Lucey JV, Costa DC, Blanes T, Busatto GF, Pilowsky LS, Takei N. Regional cerebral blood flow in obsessive-compulsive disordered patients at rest differential correlates with obsessive-compulsive and anxious-avoidant dimensions. Br J Psychiatry 1995;167:629-34.

27. Lucey JV, Costa DC, Adshead G, Deahl M, Busatto G, Gacinovic S, et al Brain blood flow in anxiety disorders: OCD, panic disorder with agoraphobia, and post-traumatic stress disorder on 99mTcHMPAO Single Photon Emission Tomography (SPET). Br J Psychiatry 1997; 171:346-50.

28. Crespo-Facorro B, Cabranes JA, Lopez-Ibor Alcocer MI, Paya B, Fernandez Perez C, Encinas M, et al. Regional cerebral blood flow in obsessive-compulsive patients with and without a chronic tic disorder. A SPECT study. Eur Arch Psychiatry Clin Neurosci 1999;249:156-61.

29. Busatto GF, Zamignani DR, Buchpiguel CA, Garrido GE, Glabus MF, Rocha ET, et al. A voxel-based investigation of regional cerebral blood flow abnormalities in obsessive-compulsive disorder using single photon emission computed tomography (SPECT). Psychiatry Res 2000;99:15-27.

30. Stein DJ, Van Heerden B, Wessels CJ, Van Kradenburg J, Warwick J, Wasserman HJ. Single photon emission computed tomography of the brain with Tc-99m HMPAO during sumatriptan challenge in obsessivecompulsive disorder: investigating the functional role of the serotonin autoreceptor. Prog Neuropsychopharmacol Biol Psychiatry 1999;23:1079-99.

31. Hoehn-Saric R, Pearlson GD, Harris GJ, Machlin SR, Camargo EE. Effects of fluoxetine on regional cerebral blood flow in obsessive-compulsive patients. Am J Psychiatry 1991;148(9):1243-5

Correspondência: Paulo Dalgalarrondo

Departamento de Psicologia Médica e Psiquiatria - FCM - Unicamp

Caixa Postal 1170 - CEP 13083-970 Campinas, SP 
Rev Bras Psiquiatr 2001;23(Supl I):61-4

Neuroimagem no TOC - p. 24-27

Figura 1 - SPECT do paciente M., antes do tratamento. Observar a hiperativação frontal, em comparação com a região occipital.

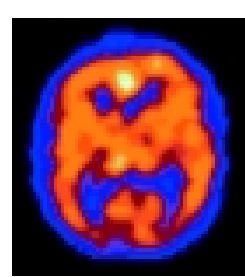

Figura 2 - SPECT do paciente da figura 1 , após tratamento com clomipramina por 6 meses. Observar o "equilíbrio de perfusão" entre todas as regiões mostradas.

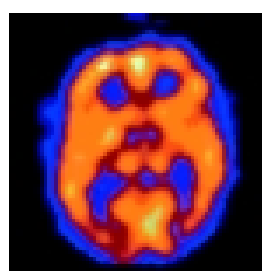

Figura 3 - SPECT de um controle saudável. Observar o "equilíbrio de perfusão" entre todas as regiões mostradas.

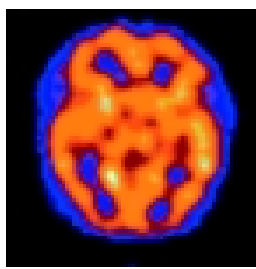

